\title{
GENERALIZED INTEGRALS WITH RESPECT TO FUNCTIONS OF BOUNDED VARIATION
}

\author{
R. L. JEFFERY
}

Introduction. A considerable literature has grown up around the analysis of the structure of a function in terms of its derivative, and the structure of functions $F(x)$ which are integrals of various kinds. Some of this relates to derivatives and integrals of $F(x)$ with respect to functions of bounded variation $\omega(x)(\mathbf{1}-\mathbf{6})$ or, in the case of a paper by Ward (4), with respect to a function of generalized bounded variation in the restricted sense. While functions of bounded variation have at most a denumerable set of discontinuities, yet this set can be everywhere dense, and in the studies to which we refer a consideration of these discontinuities enters, sometimes in a complicated way. In the present paper results are obtained without reference to the values of $F(x)$ or $\omega(x)$ at the points of discontinuity of $\omega$. The results lead to a descriptive definition of a Lebesgue-Stieltjes integral of a function with respect to $\omega$ and a descriptive definition of a generalized integral with respect to $\omega$. The latter involves functions $F(x)$ which are generalized absolutely continuous relatively to $\omega$.

Because $\omega(x)$ can be written as the difference of two non-decreasing functions there is no loss of generality in taking $\omega(x)$ to be non-decreasing. We shall consider a function $\omega(x)$ on a closed interval $[a, b]$ with the understanding that $w(x)=\omega(a), x<a, \omega(x)=\omega(b), x>b$. With $\omega(x)$ given we shall denote by $\mathfrak{U}$ a class of functions $F(x)$ defined at the points of continuity of $\omega(x)$ on $[a, b]$. Furthermore, if $\mathbb{E}$ is the set over which $\omega$ is continuous, then $F(x)$ is continuous over $\mathbb{E}$ at points of $\mathbb{E}$, and if $x_{0}$ is a point of discontinuity of $\omega$ then $F(x)$ tends to a limit as $x$ tends to $x_{0}+$ and to $x_{0}-, x \in \mathbb{C}$. These limits will be denoted by $F\left(x_{0}+\right), F\left(x_{0}-\right)$. Also $F(x)=F(a+)$ for $x<a$ and $F(x)=F(b-)$ for $b>a . F(x)$ may, or may not, be defined at points of discontinuity of $\omega$.

1. The $\omega$-measure of a set $E$ on $[a, b]$. Let $\left(a^{\prime}, b^{\prime}\right)$ be an open interval on $[a, b]$. The $\omega$-measure of $\left(a^{\prime}, b^{\prime}\right)$ is $\omega\left(b^{\prime}-\right)-\omega\left(a^{\prime}+\right)$, and is denoted by $\left|\left(a^{\prime}, b^{\prime}\right)\right|_{\omega}$. Let $E$ be any set on $[a, b]$. Let $\alpha_{1}, \alpha_{2}, \ldots$ be a set of non-overlapping open intervals containing $E$. The outer $\omega$-measure of the set $E$ is the infimum of $\Sigma\left|\alpha_{i}\right|_{\omega}$ for all such sets of open intervals. This outer measure is denoted by $|E|_{\omega}{ }^{0}$. Let $\widetilde{E}$ be the complement of the set $E$. If for $\epsilon>0$ there exists a set of non-overlapping open intervals $\alpha=\alpha_{1}+\alpha_{2}+\ldots, \alpha \supset E$ and a similar set $\beta \supset \widetilde{E}$ for which $|\alpha \beta|{ }_{\omega}{ }^{0}<\epsilon$ then the set $E$ is said to be $\omega$-measurable. The $\omega$-measure of $E$, denoted by $|E|_{\omega}$, is equal to $|E|_{\omega}{ }^{0}$.

Received October 21, 1957. 
Lemma 1. If $\alpha=\alpha_{1}+\alpha_{2}+\ldots$ is a set of non-overlapping open intervals $\alpha_{1}, \alpha_{2}, \ldots$ then $\alpha$ is $\omega$-measurable.

Because $\omega(x)$ is $B V$ it follows that $\Sigma\left|\alpha_{i}\right|_{\omega}$ converges. Hence for $\epsilon>0$ there exists an integer $n_{0}$ such that for $n>n_{0}$,

$$
\sum_{n+1}^{\infty}\left|\alpha_{i}\right|_{\omega}<\epsilon
$$

Fix $n>n_{0}$. Let $\alpha_{i}=\left(a_{i}, b_{i}\right), i \leqslant n$. Take $a_{i}{ }^{\prime}, b_{i}{ }^{\prime}$ with $a_{i}<a_{i}{ }^{\prime}<b_{i}{ }^{\prime}<b_{i}$ and such that

$$
\sum_{i=1}^{n}\left\{\left|\left(a_{i}, a_{i}^{\prime}\right)\right|_{\omega}+\left|\left(b_{i}^{\prime}, b_{i}\right)\right|_{\omega}\right\}<\epsilon .
$$

Let $\beta$ be the open set complementary to the finite set of closed intervals. $\left[a_{1}{ }^{\prime}, b_{i}{ }^{\prime}\right], \ldots,\left[a_{n}{ }^{\prime}, b_{n}{ }^{\prime}\right]$. Then $\beta \supset \widetilde{E}$ and

$$
|\alpha \beta|_{\omega}^{0}=\sum_{n+1}^{\infty}\left|\alpha_{i}\right|_{\omega}+\sum_{i=1}^{n}\left\{\left|\left(a_{i}, a_{i}^{\prime}\right)\right|_{\omega}+\left|\left(b_{i}^{\prime}, b_{i}\right)\right|_{\omega}\right\}<2 \epsilon .
$$

Because $\epsilon$ is arbitrary it follows that $\alpha$ satisfies the definition of measurability.

Lemma 2. Let $E$ be any set on $[a, b]$. Let each point $x \in E$ be the left hand end point of a set of intervals $\left(x, x+h_{i}\right)$ for which $h_{i} \rightarrow 0$ as $i \rightarrow \infty$. Let $\mathfrak{F}$ denote the set of intervals thus associated with the set $E$. Let $\epsilon>0$ be given.

There exists a finite non-overlapping set $\Delta_{i}$ of the intervals of $\mathfrak{F}$ for which

$$
\sum_{i=1}^{n}\left|\Delta_{i}\right|_{\omega}<|E|_{\omega}^{0}+\epsilon, \quad \sum_{i=1}^{n}\left|\Delta_{i} E\right|_{\omega}^{0}>|E|_{\omega}^{0}-\epsilon .
$$

Let $\eta>0$ be given. Put $E$ in a set of open intervals $\alpha_{1}, \alpha_{2}, \ldots$ in such a way that $\Sigma\left|\alpha_{i}\right|_{\omega}<|E|_{\omega}{ }^{0}+\eta$. Now let $\alpha$ be a finite set of the intervals $\alpha_{1}, \alpha_{2}, \ldots, \alpha=\alpha_{1}+\alpha_{2}+\ldots+\alpha_{n}$ where $n$ is sufficiently great to insure that $|\alpha E|_{\omega}{ }^{0}>|E|_{\omega}{ }^{0}-\eta$. Let $\alpha_{i}=\left(a_{i}, b_{i}\right)$. Let $\eta^{\prime}$ be so fixed that if $b_{i}{ }^{\prime}$ is on $\left(a_{i}, b_{i}\right), a_{i}<b_{i}{ }^{\prime}<b_{i}$ and $b_{i}-b_{i}{ }^{\prime}<\eta^{\prime}$ then

$$
\sum_{i=1}^{n}\left|E\left(b_{i}^{\prime} b_{i}\right)\right|_{\omega}^{0}<\eta \text {. }
$$

Let $E_{\delta}$ be the points of $E \alpha$ which are such that if $x$ is in $E_{\delta}$ then there is an interval $(x, x+h)$ of $\mathfrak{F}$ with $h>\delta$ and $x, x+h$ on the same interval of the set $\alpha$. If $\delta_{2}<\delta_{1}$ then

$$
E_{\delta_{2}} \supset E_{\delta_{1}} \text {. }
$$

Hence $\left|E_{\delta}\right|_{\omega}{ }^{0} \rightarrow|E \alpha|_{\omega}{ }^{0}$ as $\delta \rightarrow 0$. Fix $\delta<\eta^{\prime}$ and sufficiently near zero to insure that

$$
\left|E_{\delta}\right|_{\omega}^{0}>|E \alpha|_{\omega}^{0}-\eta>|E|_{\omega}^{0}-2 \eta \text {. }
$$

Now consider the interval $\alpha_{1}=\left(a_{1}, b_{1}\right)$. There is a point $x_{1}{ }^{\prime} \geqslant a_{1}$ which is (a) the first point of $E_{\delta}$ to the right of $a_{1}$ or (b) the infimum of points of $E_{\delta}$ on 
$\left(a_{1}, b_{1}\right)$. For case $(a)$ let $x_{1}^{\prime}=x_{1}$ and let $\left(x_{1}, x_{1}+h_{1}\right)$ be an interval of $\mathfrak{F}$ on $\left(a_{1}, b_{1}\right)$ with $h_{1}>\delta$. For case (b) take $x_{1}$ a point of $E_{\delta}$ and such that

$$
\left|E\left(x_{1}^{\prime}, x_{1}\right)\right|_{\omega}^{0}<\epsilon_{1}
$$

where $\epsilon_{1}$ is the first member of a sequence of positive numbers $\epsilon_{1}, \epsilon_{2}, \ldots$ for which $\Sigma \epsilon_{i}<\eta / n$, and take $\left(x_{1}, x_{1}+h_{1}\right)$ an interval of $\mathfrak{F}$ with $h_{1}>\delta$.

In the foregoing replace $a_{1}$ by $x_{1}+h_{1}$ and arrive at $x_{2}{ }^{\prime} \geqslant x_{1}+h_{1}$ satisfying (a) or (b) and an interval $\left(x_{2}, x_{2}+h_{2}\right)$ of $\mathfrak{F}$ on $\left(a_{1}, b_{1}\right)$ corresponding to $\left(x_{1}, x_{1}+h_{1}\right)$ with $h_{2}>\delta$. If case (b) holds choose $x_{2}$ so that

$$
\left|E\left(x_{2}^{\prime}, x_{2}\right)\right|_{\omega}^{0}<\epsilon_{2} \text {. }
$$

Since each $h_{1}>\delta$, and $\delta<\eta^{\prime}$ this procedure can be continued to get a finite non-overlapping set of intervals $\left[x_{1}, x_{1}+h_{1}\right],\left[x_{2}, x_{2}+h_{2}\right], \ldots,\left(x_{m}, x_{m}+h_{m}\right)$ of the set $\mathfrak{F}$ for which no points of $\alpha_{1} E_{\delta}$ are to the right of $x_{m}+h_{m}$ or

$$
b_{1}-\left(x_{m}+h_{m}\right)<\eta^{\prime} \text {. }
$$

Also the intervals $\left(x_{i}, x_{i}+h_{i}\right), i=1,2, \ldots, m$, have been so chosen that

$$
\sum_{i=1}^{m}\left|E\left(x_{i}^{\prime}, x_{i}\right)\right|_{\omega}<\sum \epsilon_{i}<\frac{\eta}{n} .
$$

This procedure can be repeated for each of the remaining intervals $\alpha_{2}, \alpha_{3}, \ldots$, $\alpha_{n}$ of the set $\alpha$.

From (1), (5), (6) and the relations similar to (5) and (6) for the intervals $\alpha_{2}, \ldots, \alpha_{n}$ it follows that the total set $\Delta_{i}=\left(x_{i}, x_{i}+h_{i}\right)$ obtained by this process are on $\alpha$, are non-overlapping and

$$
\sum\left|E_{\omega} \Delta_{i}\right|>\left|\alpha E_{\delta}\right|-2 \eta \text {. }
$$

This, with (2) and the fact that $E_{\delta}$ is on $\alpha$ and $\Delta_{i} E_{\delta} \subset \Delta_{i} E$, gives $\Sigma \Delta_{i} E>|E|_{\omega}{ }^{0}$ $-4 \eta$. Because $\Delta_{i}$ is on $\alpha$ and because $\eta$ is arbitrary the lemma follows.

Definition 1. A function $F(x)$ defined on $[a, b]$ and in class $\mathfrak{U}$ is absolutely continuous relative to $\omega, A C-\omega$ if for $\epsilon>0$ there exists $\delta>0$ such that for any set of non-overlapping intervals $\left(x_{i}, x_{i}{ }^{\prime}\right)$ on $[a, b]$ with $\Sigma\left\{\alpha\left(x_{i}{ }^{\prime}+\right)\right.$ $\left.-\alpha\left(x_{i}-\right)\right\}<\delta$ the relation $\Sigma\left|F\left(x_{i}{ }^{\prime}+\right)-F\left(x_{i}-\right)\right|<\epsilon$ is satisfied.

2. The derivatives of $F(x)$ with respect to $\omega$. Let $F(x)$ be a function in class $\mathfrak{U}$. Define the function $\psi(x, h)$ by the relation

$$
\psi(x, h)= \begin{cases}\frac{F(x+h)-F(x-)}{\omega(x+h)-\omega(x-)}, & h>0, \omega(x+h)-\omega(x-) \neq 0, \\ \frac{F(x+h)-F(x+)}{\omega(x+h)-\omega(x+)}, & h<0, \omega(x+h)-\omega(x+) \neq 0, \\ 0, & \omega(x+h)-\omega(x \pm)=0,\end{cases}
$$

for points of continuity $x+h$ of $\omega$. If $\psi(x, h)$ tends to a limit as $h \rightarrow 0$ this limit is the derivative of $F(x)$ with respect to $\omega(x), D_{\omega} F$. The upper and lower limits of this ratio are the upper and lower derived numbers of $F(x)$ 
with respect to $\omega$. It is to be noted that $D_{\omega} F$ exists at points of discontinuity of $\omega$ even when $F(x)$ is not defined at such a point.

Definition 2. Let $f(x)$ be defined on an $\omega$-measurable set $E$ on $[a, b]$. Let $e_{a}$ be the part of $E$ for which $f(x)<a$. If $e_{a}$ is $\omega$-measurable for every real number $a$ then $f$ is w-measurable on $E$.

Definition 3. Let $f(x)$ be $\omega$-measurable on an $\omega$-measurable set $E$. Let $\left(l_{i-1}, l_{i}\right)$ be a subdivision of the range of $f$ on $E$. Let $e_{i}$ be the points of $E$ for which $l_{i-1} \leqslant f<l_{i}$. If $\Sigma l_{i-1}\left|e_{i}\right|_{\omega}$ tends to a limit as the supremum of $l_{i}-l_{i-1} \rightarrow 0$ then this limit is the Lebesgue-Stieltjes integral of $f(x)$ over the set $E$.

If $x$ is a point on $[a, b]$ and $f(x)$ is $\omega$-measurable on $[a, b]$ then

$$
F(x)=\int_{a}^{x} f(x) d \omega
$$

is absolutely continuous relatively to $\omega$. Also $F(x)$ is in class $\mathfrak{U}$ and $D_{\omega} F=f$ except for a set of $\omega$-measure zero. This definition of a Lebesgue-Stieltjes integral is the usual one and the stated properties may be proved in the usual way.

\section{Properties of functions in class $\mathfrak{U}$.}

Definition 4. Let $F(x)$ be a function in class $\mathfrak{U}$. The set $[x, F(x)]$ is the union of the graphs $[x, F(x+)]$ and $[x, F(x-)]$. For any interval of continuity $[x, F(x)]$ is thus the graph of $F(x)$ in the usual sense.

Theorem 1. Let $F(x)$ and $G(x)$ be two functions in class $\mathfrak{u}$. If $F$ and $G$ are $A C-\omega$ and if $D_{\omega} F=D_{\omega} G$, except at a set of $\omega$-measure zero, then the sets $[x, F(x)],[x, G(x)]$ are identical or one is a translation parallel to the $y$-axis of the other.

Set $H(x)=F(x)-G(x)$. Then $H$ is $A C-\omega$ and $D_{\omega} H=0$ except for a set of $\omega$-measure zero. At a point of discontinuity $x_{0}$ of $\omega(x), H\left(x_{0}-\right)=$ $H\left(x_{0}+\right)$. For otherwise

$$
\lim _{h \rightarrow 0} \frac{H\left(x_{0}+\right)-H\left(x_{0} \pm\right)}{\omega\left(x_{0}+h\right)-\omega\left(x_{0} \pm\right)} \neq 0
$$

and it follows that $D_{\omega} H \neq 0$ on a set of $\omega$-measure greater than zero, which is a contradiction. If at points of discontinuity of $\omega$ we set $H(x)=H(x+)$ $=H(x-)$ then $H(x)$ is continuous on $[a, b]$. We now prove that $H(x)$ is constant on $[a, b]$.

Let $E$ be the set on $[a, b]$ at which $D_{\omega} H=0$. Then $|E|_{\omega}=|[a, b]|_{\omega}$. If $x$, is a point of $E$ and $\epsilon>0$ is given there is a sequence of intervals $\left[x, x+h_{i}\right]$, $h_{i}>0, h_{i} \rightarrow 0$ as $i \rightarrow \infty$ such that

$$
\left|\frac{H\left(x+h_{i}\right)-H(x-)}{\omega\left(x+h_{i}\right)-\omega(x-)}\right|<\epsilon .
$$


By Lemma 2 there is a finite set $\Delta_{i}$ of these intervals associated with the set $E$ such that

$$
\left.|\Sigma| \Delta_{i}\right|_{\omega}-\left.|[a, b]| \omega|<\epsilon, \quad \Sigma| \bar{\Delta}_{j}\right|_{\omega}<\epsilon,
$$

where $\bar{\Delta}_{j}$ is the finite set of intervals complementary to the set $\Delta_{i}$. It follows from the second member of (2) and the fact that $H(x)$ is $A C-\omega$ that if $\eta$ is given then $\epsilon$ can be so fixed that if $\left[x_{j}, x_{j}{ }^{\prime}\right]$ are the intervals of $\bar{\Delta}_{j}$ then

$$
\Sigma\left|H\left(x_{j}^{\prime}\right)-H\left(x_{j}\right)\right|<\eta \text {. }
$$

It follows from (1) that for the intervals $\Delta_{i}$

$$
\Sigma\left|H\left(x_{i}{ }^{\prime}\right)-H\left(x_{i}\right)\right| \leqslant \epsilon M
$$

where $M$ depends on the function of bounded variation $\omega(x)$. It then follows that $|H(b)-H(a)|<\epsilon M+\eta$. Because $\epsilon$ and $\eta$ are arbitrary, $\epsilon$ fixed after $\eta$, it follows that $F(b)=F(a)$. If $a<x<b$ it can be shown in the same way that $H(x)=H(a)$. Hence $H(x)$ is constant on $[a, b]$.

It now follows that $F(x)-G(x)=C$, a constant, at points where both functions are continuous, that is, at the points of continuity of $\omega$. Furthermore, at points $x_{0}$ of discontinuity of $\omega$,

$$
\lim _{x \rightarrow x_{0} \pm 0}[F(x)-G(x)]=C,
$$

$x$ a point of continuity of $\omega$, from which it follows that at points of discontinuity of $\omega$ the jumps, if any, of $F$ and $G$ are the same and in the same direction. It then follows that the sets $[x, F(x)],[x, G(x)]$ are identical or one is a translation parallel to the $y$-axis of the other.

Theorem 2. If $F(x)$ is in class $\mathfrak{u}$ and is $A C-\omega$ and if $D_{\omega} F=f(x)$, except for a set of $\omega$-measure zero, then if $a$ and $x$ are points of continuity of $\omega$

$$
F(x)-F(a)=\int_{a}^{x} f(x) d \omega .
$$

Let

$$
G(x)=\int_{a}^{x} f(x) d \omega .
$$

Then $G(x)$ is in class $\mathfrak{u}$, is $A C-\omega$, and $D_{\omega} G=f(x)$ except for a set of $x$ of $\omega$-measure zero. Hence $G$ and $F$ satisfy the conditions of Theorem 1, and, except at points of discontinuity of $\omega$

$$
F(x)-G(x)=C \text {. }
$$

Since $G(a)=0$ it follows that $C=F(a)$ and

$$
F(x)-F(a)=G(x)=\int_{a}^{x} f(x) d \omega .
$$

This theorem gives point to the following definition: 
Definition 5. Let $f(x)$ be defined on $[a, b]$ and be measurable relative to $\omega$. Let $F(x)$ be a function in class $\mathfrak{U}$ which is $A C-\omega$ and such that $D_{\omega} F=f(x)$ except possibly for a set of $\omega$-measure zero. If the series $\Sigma\left|f\left(x_{i}\right)\left\{\omega\left(x_{i}+\right)-\omega\left(x_{i}-\right)\right\}\right|$, where $x_{i}, i=1,2, \ldots$, are the points of discontinuity of $\omega$, converges and if $F\left(x_{i}+\right)-F\left(x_{i}-\right)=f\left(x_{i}\right)\left\{\omega\left(x_{i}+\right)-\omega\left(x_{i}-\right)\right\}$ then $F(x)$ is the Lebesgue-Stieltjes integral of $f(x)$ :

$$
F(x)-F(a)=\int_{a}^{x} f(t) d \omega(t)
$$

Definition 6. A function $F(x)$ is generalized absolutely continuous with respect to $\omega, A C G-\omega$, over $[a, b]$ if this interval is the sum of a denumerable sequence of closed sets over each of which $F(x)$ is $A C-\omega$.

Theorem 3. Let $F(x)$ and $G(x)$ be two functions in class $\mathfrak{U}$ each of which is $A C G-\omega$ on $[a, b]$, and such that $D_{\omega} F=D_{\omega} F$ except for a set of $\omega$-measure zero. Then the sets $[x, F(x)],[x, G(x)]$ are identical or one is a translation parallel to the $y$-axis of the other.

As in Theorem 1, let $H(x)=F(x)-G(x)$ at points of continuity of $\omega(x)$. Then $D_{\omega} H=0$ except for set of $\omega$-measure zero, and it follows as before that $H\left(x_{0}-\right)=H\left(x_{0}+\right)$ at points of discontinuity of $\omega$. Hence if $H\left(x_{0}\right)$ is equal to this common value then $H(x)$ is continuous on $[a, b]$. We now have $H(x)$ continuous and $A C G-\omega$ on $[a, b]$ and $D_{\omega} H=0$ except for a set of $\omega$-measure zero. We show that $H(x)$ is constant on $[a, b]$.

Because $F$ and $G$ are both $A C G-\omega$ on $[a, b]$ it follows that

$$
[a, b]=\sum E_{i}^{1}, \quad[a, b]=\sum E_{j}^{2}, \quad \text { where } E_{i}^{1}, E_{j}^{2} \text { are closed, }
$$

where $F$ is $A C-\omega$ over each $E_{i}{ }^{1}$ and $G$ is $A C-\omega$ over each $E_{j}{ }^{2}$. Hence $H(x)$ is $A C-\omega$ over each of the sets $E_{i}{ }^{1} E_{j}{ }^{2}, i, j=1,2, \ldots$ This is a denumerable sequence $\xi_{1}, \xi_{2}, \ldots$ of closed sets which cover $[a, b]$. Let $E$ be the set on $[a, b]$ which is such that if $x \in E$ then in every interval $\omega$ with $x$ as an interior point $H(x)$ fails to be constant. The set $E$ is closed. It then follows from Baire's theorem that there is an integer $n$ and an interval $[l, m]$ such that $E[l, m]$ is not empty, $E[l, m]=\xi_{n}[l, m]=e$. The set $e$ is closed and $H$ is $A C-\omega$ over $e$. Hence, if $\epsilon$ is given, there exists $\delta$ such that if $\left(x_{i} x_{i}{ }^{\prime}\right)$ is a set of non-overlapping intervals on $[l, m]$ with $x_{i}, x_{i}{ }^{\prime}$ points of $e$ and with $\Sigma\left|\left(x_{i}, x_{i}{ }^{\prime}\right)\right|_{\omega}<\delta$ then $\Sigma\left|H\left(x_{i}{ }^{\prime}\right)-H\left(x_{i}\right)\right|<\epsilon$. Now let $\left(x_{j}, x_{j}{ }^{\prime}\right)$ be any set of non-overlapping intervals on $[l, m]$ with $\Sigma\left|\left(x_{j}, x_{j}^{\prime}\right)\right|_{\omega}<\delta$. If there are points of $e$ on $\left[x_{j}, x_{j}^{\prime}\right]$ let $\bar{x}_{j}$ be the first point of $e$ to the right of $x_{j}, \bar{x}_{j}=x_{j}$ if $x_{j} \in e$. Let $\bar{x}_{j}^{\prime}$ be the first point of $e$ to the left of $x_{j}{ }^{\prime}, \bar{x}_{j}^{\prime}=x_{j}{ }^{\prime}$ if $x_{j}^{\prime} \in e$. Because $H(x)$ is continuous, and constant on intervals of $[l, m]$ which are complementary to the set $e=\xi_{n}[l, m]$, it follows that $H(x)$ is constant on $\left[x_{j}, \bar{x}_{j}\right]$ and on $\left[\bar{x}_{j}{ }^{\prime}, x_{j}{ }^{\prime}\right]$. We now have $H\left(\bar{x}_{j}\right)-H\left(x_{j}\right)=H\left(x_{j}{ }^{\prime}\right)-H\left(\bar{x}_{j}{ }^{\prime}\right)=0$ and 


$$
\begin{aligned}
\sum\left|H\left(x_{j}^{\prime}\right)-H\left(x_{j}\right)\right| \leqslant & \sum\left|H\left(\bar{x}_{j}\right)-H\left(x_{j}\right)\right|+ \\
& \sum\left|H\left(\overline{x_{j}^{\prime}}\right)-H\left(\bar{x}_{j}\right)\right|+\sum\left|H\left(x_{j}^{\prime}\right)-H\left(\overline{x_{j}^{\prime}}\right)\right| \\
\leqslant & \sum\left|H\left(\overline{x_{j}^{\prime}}\right)-H\left(\bar{x}_{j}\right)\right|<\epsilon .
\end{aligned}
$$

The last relation follows because $\bar{x}_{j}, \bar{x}_{j}{ }^{\prime}$ are points of $\xi_{n}$ and $\Sigma\left|\left(\bar{x}_{j}, \bar{x}_{j}{ }^{\prime}\right)\right|_{\omega}$ $\leqslant \Sigma\left|\left(x_{j}, x_{j}{ }^{\prime}\right)\right|_{\omega}<\delta$. Then, because $\left(x_{j}, x_{j}{ }^{\prime}\right)$ is any set of intervals on $[l, m]$ it follows that $H(x)$ is $A C-\omega$ on $[l, m]$. Because $D_{\omega} H=0$ except for a set of $\omega$-measure zero it now follows from Theorem 1 that $H(x)$ is constant on $[l, m]$. Consequently, there are no points of $E$ on $[l, m]$. But this contradicts the fact that $E$ is not empty. Hence the set $E$ is empty. It now follows that every point of $[a, b]$ is interior to an interval on which $H(x)$ is constant. By the Heine-Borel theorem there is a finite set of intervals covering $[a, b]$ on each of which $H(x)$ is constant. Then, because $H(x)$ is continuous it easily follows that $H(x)$ is constant on $[a, b]$. The proof of Theorem 3 may now be completed as in Theorem 1.

Theorem 3. Let $F(x)$ be a function in class $\mathfrak{U}$ which is $A C G-\omega$ on $[a, b]$. Let $f(x)$ be a $\omega$-measurable function on $(a, b)$ and let $D_{\omega} F=f$ except for at most $a$ set of $\omega$-measure zero. Then $F(b+)-F(a-)$ can be determined in at most a denumerable set of operations.

Lemma 2. Let $E$ be a closed set on $[a, b]$. If $F(x)$ satisfies the conditions of Theorem 3 there is an interval $[l, m]$ on $[a, b]$ such that $E[l, m]$ is not empty, such that $D_{\omega} F$ is Lebesgue-Stieltjes integrable with respect to $\omega$ over $E[l, m]$, and such that if $\left[\alpha_{i}, \beta_{i}\right]$ are the intervals on $[l, m]$ contiguous to $E[l, m]$, then $\Sigma \mid F\left(\beta_{i}+\right)$ $-F\left(\alpha_{i}-\right) \mid$ converges.

Let $[a, b]=\xi_{1}+\xi_{2}+\ldots$ where each $\xi_{n}$ is closed and $F$ is $A C-\omega$ over $\supset_{n}$. There is then an integer $n$ and an interval $[l, m]$ such that $E[l, m]$ $=\xi_{n}[l, m]=e$. We now turn our attention to the function $F(x)$ on $[l, m]$. $F$ is $A C-\omega$ over $e$. It then easily follows that there exists a positive number $M$ such that for any set of non-overlapping intervals $\left(x_{i}, x_{i}{ }^{\prime}\right)$ on $[a, b]$ with $x_{i}, x_{i}{ }^{\prime}$ points of $e$ the relation $\Sigma\left|F\left(x_{i}{ }^{\prime}+\right)-F\left(x_{i}-\right)\right|<M$ holds. We use this fact in showing that $\left|D_{\omega} F\right|=|f|$ is Lebesgue-Stieltjes integrable with respect to $\omega$ over $e$.Let $\left(l_{i-1}, l_{i}\right), i=1,2, \ldots$, be a subdivision of the range $[0, \infty]$. Let $e_{i}=E\left(l_{i-1}<|f| \leqslant l_{i}, x \in e\right), i>1, e_{0}=E\left(l_{0} \leqslant f \leqslant l_{1}\right)$. Suppose that $\Sigma l_{i-1}\left|e_{i}\right|_{\omega}$ diverges. Fix $n$ so that

$$
\sum_{i=1}^{n} l_{i-1}\left|e_{i}\right|_{\omega}>2 M
$$

If $x \in e_{i}$ there is a sequence of intervals $\left(x, x+h_{i}\right), h_{i}>0, h_{i} \rightarrow 0$ such that

$$
\frac{\left|F\left(x+h_{i}\right)-F(x-)\right|}{\left|\alpha\left(x+h_{i}\right)-\alpha(x-)\right|}>l_{i-1} \text {. }
$$


Let $\epsilon_{0}, \epsilon_{1}, \epsilon_{2}, \ldots$ be a sequence of positive numbers with $\epsilon_{n} \rightarrow 0$. By Lemma 2 there is a finite set $\Delta_{i}{ }^{0}$ of non-overlapping intervals of the set associated with the set $e_{0}$ by means of (3) for which

$$
\sum\left|\Delta_{i}{ }^{0}\right|_{\omega}>\left|e_{0}\right|_{\omega}-\epsilon_{0}, \quad \sum\left|\tilde{e}_{0} \Delta_{i}{ }^{0}\right|_{\omega}<\epsilon_{0} .
$$

Because of the second relation of (4) there exists a finite set $\Delta_{i}{ }^{1}$ of the intervals associated with $e_{1}$ by means of (3) such that

$$
\sum\left|\Delta_{i}{ }^{1}\right|_{\omega}>\left|e_{1}\right|_{\omega}-\epsilon_{0}-\epsilon_{1}, \quad \sum\left|\tilde{e}_{1}\left(\Delta_{i}{ }^{0}+\Delta_{1}{ }^{1}\right)\right|_{\omega}<\epsilon_{0}+\epsilon_{1},
$$

and $\Sigma \Delta_{i}{ }^{1}$ does not overlap $\Sigma \Delta_{i}{ }^{0}$. If this process is continued there is obtained a set of intervals $\Delta_{i}{ }^{k}$, which do not overlap the set $\Sigma \Delta_{i}{ }^{0}+\Sigma \Delta_{i}{ }^{1}+\ldots+\Sigma \Delta_{i}{ }^{k-1}$, such that

$$
\sum\left|\Delta_{i}^{k}\right|_{\omega}>\left|e_{k}\right|_{\alpha}-\epsilon_{0}-\epsilon_{1}-\ldots-\epsilon_{k},
$$

and

$$
\sum\left|\tilde{e}_{k}\left(\Delta_{i}{ }^{0}+\Delta_{i}{ }^{1}+\ldots+\Delta_{i}{ }^{k}\right)\right|_{\omega}<\epsilon_{0}+\epsilon_{1}+\ldots+\epsilon_{k} .
$$

Also, because of (3), it is true that if $\left(x_{i}, x_{i}{ }^{\prime}\right)$ are the intervals of the set $\Delta_{i}{ }^{k}$ then

$$
\frac{F\left(x_{i}^{\prime}+\right)-F\left(x_{i}-\right)}{\alpha\left(x_{i}{ }^{\prime}+\right)-\alpha\left(x_{i}-\right)}>l_{k-1}
$$

and

$$
\sum\left\{F\left(x_{i}^{\prime}+\right)-F\left(x_{i}-\right)\right\}>l_{k-1}\left(\left|e_{k}\right|_{\omega}-\epsilon_{0}-\epsilon_{1}-\ldots-\epsilon_{k}\right) .
$$

Combining all the sets $\Delta_{i}{ }^{k}$ into a single set $\Delta_{i}=\left(x_{i}, x_{i}{ }^{\prime}\right)$ and summing over this set we get

$$
\sum\left|F\left(x_{i}{ }^{\prime}+\right)-F\left(x_{i}-\right)\right|>\sum_{k=1}^{n} l_{k-1}\left|e_{k}\right|_{\omega}-n l_{0} \epsilon_{0}-(n-1) l_{1} \epsilon_{1}-\ldots-l_{n-1 \epsilon_{n}} .
$$

The first sum on the right is greater than $2 M$. The numbers $\epsilon_{0}, \epsilon_{1}, \ldots, \epsilon_{n}$ are independent of $n$, and independent of the numbers $l_{0}, l_{1}, \ldots, l_{n-1}$. Hence the left side is not less than 2M. But $x_{i}, x_{i}{ }^{\prime}$ are points of $e$ which compels the left side to be less than $M$. Thus there is a contradiction and we may conclude that $\Sigma l_{i-1}\left|e_{i}\right|_{\omega}$ converges. It then follows that $\left|D_{\omega} F\right|$ is LebesgueStieltjes integrable with respect to $\omega$ over $e$.

Let $\left(\alpha_{i}, \beta_{i}\right)$ be the intervals on $[l, m]$ complementary to $e$. Let $e>0$ be given. Fix $n_{0}$ with

$$
\sum_{n_{0}+1}^{\infty}\left|F\left(\beta_{i}+\right)-F\left(\alpha_{i}-\right)\right|<\epsilon
$$

Take $\left(l_{i-1}, l_{i}\right)$ a subdivision of $(-\infty, \infty)$. Let $e_{i}, e_{i}{ }^{\prime}$ be respectively the parts of $e$ for which $l_{i-1}<D_{\omega} F \leqslant l_{i}, l_{i-1} \leqslant D_{\omega} F<l_{i}$. Let the subdivision $\left(l_{i-1}, l_{i}\right)$ and the integer $n_{1}$ be such that

$$
\sum_{-n_{1}}^{n_{1}} l_{i-1}\left|e_{i}\right|_{\omega}, \quad \sum_{-n_{1}}^{n_{1}} l_{i}\left|e_{i}\right|_{\omega}
$$


differ from the Lebesgue-Stieltjes interval of $D_{\omega} F$ over $e$ by not more than $\epsilon$. By working as before with the sets $e_{i}$ we can get a set of intervals $\Delta_{i}$ with end points $x_{i}, x_{i}{ }^{\prime}$ belonging to $e$ and such that the intervals $\Delta_{i}$ do not overlap the intervals $\left(\alpha_{i}, \beta_{i}\right), i=1,2, \ldots, n_{0}$, such that

$$
\sum_{i=1}^{k} F\left(x_{i}{ }^{\prime}+\right)-F\left(x_{i}-\right)>\sum_{i=1}^{k} l_{i-1}\left|e_{i}\right|_{\omega}-\epsilon>\int_{e} D_{\omega} F d_{\omega}-2 \epsilon,
$$

and such that the finite set of intervals $\bar{\Delta}_{j}$ complementary to the set $\Delta_{i}$ and the set $\left(\alpha_{i}, \beta_{i}\right), i=1,2, \ldots, n_{0}$, satisfy $\Sigma\left|\bar{\Delta}_{j}\right|_{\omega}<\delta$, from which it follows that $\Sigma\left|F\left(x_{j}^{\prime}\right)-F\left(x_{j}\right)\right|<\epsilon$. Hence

$$
\begin{aligned}
& F(m+)-F(l-)=\sum_{1}^{n_{0}}\{\left.F\left(\beta_{i}\right)-F\left(\alpha_{i}\right)\right\}+\sum\left\{F\left(x_{i}{ }^{\prime}+\right)-F\left(x_{i}-\right)\right\} \\
&+\sum\left\{F\left(x_{j}{ }^{\prime}\right)-F\left(x_{j}\right)\right\} \\
&>\sum_{1}^{\infty}\left\{F\left(\beta_{i}\right)-F\left(\alpha_{i}\right)\right\}+\int_{e} D_{\omega} F d_{\omega}-3 \epsilon .
\end{aligned}
$$

If a similar procedure is used with the sets $e_{i}{ }^{\prime}$ it may be shown that

$$
F(m+)-F(l-)<\sum_{1}^{\infty}\left\{F\left(\beta_{i}+\right)-F\left(\alpha_{i}-\right)\right\}+\int_{e} D_{\omega} F d_{\omega}+3 \epsilon .
$$

Because $\epsilon$ is arbitrary we conclude that

$$
F(m+)-F(l-)=\int_{e} D_{\omega} F d \omega+\sum\left\{F\left(\beta_{i}-\right)-F\left(\alpha_{i}+\right)\right\} .
$$

We can now state that if $E$ is any closed set on $[a, b]$ there is an interval $[l, m]$ containing points of $E$ such that $D_{\omega} F$ is summable over $E[l, m]$ and such that if $\left(\alpha_{i}, \beta_{i}\right)$ are the intervals on $[l, m]$ complementary to the set $E[l, m]$ then

$$
F(m+)-F(l-)=\int_{E[l, m]} D_{\omega} F d \omega+\sum\left\{F\left(\beta_{i}+\right)-F\left(\alpha_{i}-\right)\right\} .
$$

Let $E_{1}$ be the points of non-summability of $D_{\omega} F$ over $[a, b],\left(\alpha_{i}, \beta_{i}\right)$ the intervals complementary to $E_{1}$. If $\left(\alpha^{\prime}, \beta^{\prime}\right)$ is an interval such that $\alpha_{i}<\alpha^{\prime}$ $<\beta^{\prime}<\beta_{i}$ then $D_{\omega} F$ is Lebesgue-Stieltjes integrable over $\left[\alpha^{\prime}, \beta^{\prime}\right]$ and

$$
F\left(\beta^{\prime}+\right)-F\left(\alpha^{\prime}-\right)=\int_{\alpha^{\prime}}^{\beta^{\prime}} D_{\omega} F d \omega .
$$

Because of the continuity properties of $F$ it follows that as $\alpha^{\prime} \rightarrow \alpha_{i}, \beta^{\prime} \rightarrow \beta_{i}$

$$
F\left(\beta^{\prime}+\right)-F\left(\alpha^{\prime}-\right) \rightarrow F\left(\beta_{i}-\right)-F\left(\alpha_{i}+\right),
$$

and

$$
\begin{aligned}
F\left(\beta_{i}+\right)-F\left(\alpha_{i}-\right)=F\left(\beta_{i}-\right)-F\left(\alpha_{i}+\right) & \\
& +\int_{\beta_{i}} D_{\omega} F d \omega-\int_{\alpha_{i}} D_{\omega} F d \omega .
\end{aligned}
$$


Thus $F\left(\beta_{i}+\right)-F\left(\alpha_{i}-\right)$ is determined for all intervals $\left(\alpha_{i}, \beta_{i}\right)$ contiguous to the set $E_{1}$. Now let $E_{2}$ be the points of $E_{1}$ which are such that if $x \in E_{2}$ there is no interval $[l, m]$ containing $x$ with $D_{\omega} F$ summable over $E_{1}[l, m]$ and $\Sigma\left\{F\left(\beta_{i}+\right)-F\left(\alpha_{i}-\right)\right\}$ converging where $\left(\alpha_{i}, \beta_{i}\right)$ are the intervals on $[l, m]$ contiguous to the set $E_{1}[l, m]$. The set $E_{2}$ is closed and, by Lemma 2 , nondense on $E_{1}$. If $\left(\alpha_{i}, \beta_{i}\right)$ are the intervals complementary to $E_{2}$ the procedure used for the intervals complementary to $E_{1}$ can now be used to obtain $F\left(\beta_{i}+\right)-F\left(\alpha_{i}-\right)$ for these intervals $\left(\alpha_{i}, \beta_{i}\right)$ complementary to $E_{2}$. This process can be continued by transfinite inductions to arrive at $F(b+)-F(a-)$ in a denumerable number of steps.

A consideration of Theorem 3 leads to the following definition.

Definition 7. Let $\omega$ be a non-decreasing function on $[a, b]$ and let $f(x)$ be defined on $[a, b]$ and be measurable relatively to $\omega$. If there exists a function $F(x)$ in class $\mathfrak{U}$ which is $A C G-\omega$ on $[a, b]$ and is such that $D_{\omega} F=f$ except for a set of $\omega$-measure zero, and for which the relations of Definition 5 are satisfied at the points of discontinuity of $\omega$, then $F(x)$ is an indefinite LebesgueStieltjes integral of $f$ with respect to $\omega$.

The descriptive definition of an integral with respect to a non-decreasing function $\omega$ given here appears to be equivalent to the constructive definition given in $(3$, p. 666). This requires proof. Another problem for investigation is that of extending the methods of the present paper to the case in which the base function $\omega$ is $V B G$.

\section{REFERENCES}

1. C. Choquet, Applications des propriétés descriptives de la fonction contingent à la théorie de variable réelle et à géometrie différentielle des variétés Cartésiennes, J. Math. pures et appl., 26 (1947), 115-226.

2. C. A. Hayes, Jr. and C. Y. Pauc, Full individual and class differentiation theorems in their relations to halo and Vitali properties, Can. J. Math., 7 (1955), 221-274.

3. R. L. Jeffery, Non-absolutely convergent integrals with respect to functions of bounded variation, Trans. Amer. Math. Soc., 34 (1932), 645-675.

4. H. Lebesgue, Leçons sur l'intégration (Paris, 1928).

5. J. Radon, Theorie und Anwendungen der absolut additiven Mengenfunktionen, Wiener Sitzungberichte, 122 (Abt. IIA) (1913), 1295-1438.

6. A. J. Ward, The Perron-Stieltjes integral, Math. Zeit., 41 (1936), 578-604.

Queen's University, Kingston 